UWO-TH-05/06

hep-th/0507275

\title{
A holographic perspective on Gubser-Mitra conjecture
}

\author{
Alex Buchel \\ Perimeter Institute for Theoretical Physics \\ Waterloo, Ontario N2J 2W9, Canada \\ Department of Applied Mathematics \\ University of Western Ontario \\ London, Ontario N6A 5B\%, Canada
}

\begin{abstract}
We point out an elementary thermodynamics fact that whenever the specific heat of a system is negative, the speed of sound in such a media is imaginary. The latter observation presents a proof of Gubser-Mitra conjecture on the relation between dynamical and thermodynamic instabilities for gravitational backgrounds with a translationary invariant horizon, provided such geometries can be interpreted as holographic duals to finite temperature gauge theories. It further identifies a tachyonic mode of the Gubser-Mitra instability (the lowest quasinormal mode of the corresponding horizon geometry) as a holographic dual to a sound wave in a dual gauge theory. As a specific example, we study sound wave propagation in Little String Theory (LST) compactified on a two-sphere. We find that at high energies (for temperatures close to the LST Hagedorn temperature) the speed of sound is purely imaginary. This implies that the lowest quasinormal mode of the finite temperature Maldacena-Nunez background is tachyonic.
\end{abstract}

July 2005 


\section{Introduction}

Gauge theory/string theory correspondence $[1,2]$ allows to rephrase complicated questions arising in string theory (gravitational) backgrounds into more intuitive field theoretical language. In certain cases this dual formulation of the problem allows for a simple solution. In this paper we discuss a problem of just this type - the GubserMitra conjecture [3].

The Gubser-Mitra conjecture ${ }^{1}$ states that gravitational backgrounds with a translationally invariant horizon (the 'black brane' solutions) develop an instability (a tachyonic mode) precisely whenever the specific heat of the black brane geometry becomes negative. In the framework of Maldacena duality gravitational backgrounds with translationary invariant horizon arise as a dual description of strongly coupled generalized ${ }^{2}$ gauge theories at finite temperature. In what follows we consider only black brane geometries of the latter type. The holographic dual of the Gubser-Mitra conjecture then implies that a finite temperature gauge theory with a negative specific heat must have a dynamical instability. It is straightforward to explicitly identify such an instability ${ }^{3}$. Indeed, consider a thermodynamic system (without chemical potential) at fixed volume and temperature $T$. Such a media propagates small momentum $(q \ll T)$ sound waves with a dispersion relation

$$
\omega(q)=v_{s} q+\mathcal{O}\left(\frac{q^{2}}{T}\right),
$$

where the speed of sound $v_{s}$ is determined from the equation of state as

$$
v_{s}^{2}=\frac{\partial P}{\partial \mathcal{E}},
$$

with $P, \mathcal{E}$ being the pressure and the energy density correspondingly. At zero chemical potential, the free energy density $\mathcal{F}$ is $\mathcal{F}=-P$. Noting that at a fixed volume $V$

$$
-\left(\frac{\partial P}{\partial T}\right)_{V}=\left(\frac{\partial \mathcal{F}}{\partial T}\right)_{V}=-S,
$$

related to the entropy density $S$, and using the specific heat $c_{V}$ definition

$$
c_{V}=\left(\frac{\partial \mathcal{E}}{\partial T}\right)_{V},
$$

\footnotetext{
${ }^{1}$ Interesting observations on Gubser-Mitra conjecture were presented in $[4,5]$.

${ }^{2}$ In some examples [6] the Maldacena duals of string theory backgrounds have a four dimensional gauge theory interpretation only at low energies.

${ }^{3}$ After completing this paper we learned that equivalent arguments were presented in [7].
} 
we find from (2)

$$
v_{s}^{2}=\frac{(\partial P / \partial T)_{V}}{(\partial \mathcal{E} / \partial T)_{V}}=\frac{S}{c_{V}} .
$$

Since ${ }^{4} S>0$, eq. (5) implies that in a media with $c_{V}<0$ the speed of sound is purely imaginary. Thus, the amplitude of a fixed momentum sound wave would increase exponentially with time, reflecting the dynamical instability of the system. Finally, as the sound wave in hot gauge theory plasma is holographically dual to the lowest quasinormal mode of the dual black brane geometry [8], we immediately conclude that it is this mode that realizes tachyonic instability of the black brane backgrounds with a negative specific heat.

In the rest of this paper we explicitly demonstrate above observation in the context of Little String Theory $[9,10]$ compactified on $S^{2}$. The extremal supergravity solution representing a large number of type IIB string theory NS5 branes wrapped on a two-cycle of the resolved conifold was discussed in $[11,12,6](\mathrm{MN})$. In the infrared MN geometry provides a holographic dual to $\mathcal{N}=1 S U(N)$ supersymmetric Yang-Mills theory in the planar limit. This supergravity solution encodes interesting non-perturbative phenomena of the four-dimensional asymptotically free gauge theories: confinement, chiral symmetry breaking, etc. The nonextremal deformation of the MN background (holographically dual to $S^{2}$ compactified LST at finite temperature) was previously discussed in [13-15]. We compute the dispersion relation for the lowest quasinormal mode in the finite temperature MN geometry, as described by (II). We find that at high energy (at temperatures close to the LST Hagedorn temperature) the speed of sound is purely imaginary. We then reproduce the speed of sound directly from the equation of state ${ }^{5}$ computation (2). This provides a highly nontrivial consistency check on our analysis.

One of the motivations to consider finite temperature deformations of the MaldacenaNunez background [6] was to study finite temperature phase transitions (confinement/deconfinement, chiral symmetry breaking/restoration) in a QCD-like theory. Unfortunately, we demonstrate here that such deformations are tachyonic ${ }^{6}$ for the MN so-

\footnotetext{
${ }^{4}$ in the gravitational dual it is proportional to the area of the horizon

${ }^{5}$ Though both Ref. $[14,15]$ propose the (different) equation of state for the finite temperature MN geometry, neither of them reproduces the correct speed of sound. We comment on this issue later in the paper.

${ }^{6}$ Strictly speaking, we establish this only at high energy. We believe that this is true at all energy scales where supergravity approximation to the full string theory is reliable.
} 
lution. The only currently known string theory background where thermal phase transitions in a QCD-like theory can be studied quantitatively is the Klebanov-Strassler geometry [16-21].

The paper is organized as follows. In the next section we derive effective five dimensional gauged supergravity action describing finite temperature deformations of the MN background at temperatures above chiral symmetry breaking. In section 3 we construct analytic solution of the nonextremal geometry holographically dual to $S^{2}$ compactified LST, in the limit of large $S^{2}$ radius at the horizon. We argue that this parameter regime corresponds to the high energy phase of the finite temperature MN solution. We motivate a new equation of state for the system and explain the difference with the previous proposals $[14,15]$. In section 4 , following [8,22], we compute the dispersion relation for the sound wave in finite temperature MN background. We conclude in section 5 .

\section{Effective action for LST on $S^{2}$}

Extremal and nonextremal solutions of the type IIB supergravity backgrounds holographically dual to $S^{2}$ compactified LST with $\mathcal{N}=1$ supersymmetry preserving twist (at the extremality) were constructed in $[11,12,6,13,15]$. In this section we present an effective five-dimensional supergravity action describing such solutions (and perturbations about them) assuming the unbroken $U(1)$ symmetry of the MN background (corresponding to the $U(1)_{R}$ symmetry of the dual low-energy $\mathcal{N}=1$ supersymmetric Yang-Mills theory). This effective action provides a consistent five-dimensional truncation of the full type IIB supergravity geometry of a large number of NS5 branes wrapped on a two-cycle of the resolved conifold ${ }^{7}$.

Consider the following type IIB supergravity ansatz. The string frame metric is

$$
d s_{10}^{\text {string }}=\hat{g}_{\mu \nu} d x^{\mu} d x^{\nu}+\omega^{2}\left(d S_{2}\right)^{2}+n^{2}\left(d \tilde{S}_{2}\right)^{2}+n^{2}\left(d \psi+\sum_{i=1}^{2} \cos \theta_{i} d \phi_{i}\right)^{2}
$$

where $\omega=\omega\left(x^{\mu}\right),\left\{\theta_{1}, \phi_{1}\right\}$ are coordinates of the round $S_{2},\left\{\theta_{2}, \phi_{2}\right\}$ are coordinates of the round $\tilde{S}_{2}, n$ is a constant. Additionally we have a 3 -form flux

$$
H_{3}=n^{2}\left(d \psi+\sum_{i=1}^{2} \cos \theta_{i} d \phi_{i}\right) \wedge\left(\sin \theta_{1} d \theta_{1} \wedge d \phi_{1}-\sin \theta_{2} d \theta_{2} \wedge d \phi_{2}\right),
$$

\footnotetext{
${ }^{7}$ Such effective action is a direct analogy of the 'chirally symmetric' action for the Klebanov-Tseytlin geometry constructed in [21].
} 
and a dilaton $\Phi=\Phi\left(x^{\mu}\right)$. Evaluating type IIB (Einstein frame) supergravity action

$$
\begin{gathered}
S_{10}=\frac{1}{16 \pi G_{10}} \int_{\mathcal{M}_{10}}\left(R_{10} \wedge \star 1-\frac{1}{2} d \Phi \wedge \star d \Phi-\frac{1}{2} e^{-\Phi} H_{3} \wedge \star H_{3}-\frac{1}{2} e^{\Phi} F_{3} \wedge \star F_{3}\right. \\
\left.-\frac{1}{4} F_{5} \wedge \star F_{5}-\frac{1}{2} C_{4} \wedge H_{3} \wedge F_{3}\right)
\end{gathered}
$$

on the ansatz (6) (17), we obtain five dimensional effective action dual to LST compactified on $S^{2}$

$$
\begin{aligned}
S_{5}= & \frac{1}{16 \pi G_{5}} \int_{\hat{\mathcal{M}}_{5}} \operatorname{vol}_{\mathcal{M}_{5}} e^{-2 \Phi} \omega^{2}\left\{\hat{R}_{5}+2 \omega^{-2} \nabla_{\mu} \omega \nabla^{\mu} \omega-8 \omega^{-1} \nabla_{\mu} \omega \nabla^{\mu} \Phi+4 \nabla_{\mu} \Phi \nabla^{\mu} \Phi\right. \\
& \left.+n^{-2}+2 \omega^{-2}-n^{2} \omega^{-4}\right\},
\end{aligned}
$$

where the greek indexes run ${ }_{1 \ldots 5} . \hat{R}_{5}$ is the 5 d Ricci scalar computed with the metric

$$
d \hat{s}_{5}^{2}=\hat{g}_{\mu \nu}(x) d x^{\mu} d x^{\nu}
$$

$G_{5}$ is a 5 d effective gravitational constant

$$
G_{5}=\frac{G_{10}}{64 \pi^{3} n^{3}}
$$

We find it convenient to rewrite the action (9) in five-dimensional Einstein frame. The latter is achieved with the following rescaling

$$
\hat{g}_{\mu \nu} \rightarrow g_{\mu \nu} \equiv e^{-4 \Phi / 3} \omega^{4 / 3} \hat{g}_{\mu \nu} .
$$

Further introducing

$$
\alpha \equiv \ln \omega,
$$

the five dimensional effective action becomes

$$
\begin{aligned}
S_{5}= & \frac{1}{16 \pi G_{5}} \int_{\mathcal{M}_{5}} \operatorname{vol}_{\mathcal{M}_{5}}\left\{R_{5}-\frac{10}{3}(\partial \alpha)^{2}-\frac{4}{3}(\partial \Phi)^{2}+\frac{8}{3} \partial \alpha \partial \Phi\right. \\
& \left.+n^{-2} e^{\frac{4}{3} \Phi-\frac{4}{3} \alpha}+2 e^{\frac{4}{3} \Phi-\frac{10}{3} \alpha}-n^{2} e^{\frac{4}{3} \Phi-\frac{16}{3} \alpha}\right\} .
\end{aligned}
$$

From the effective action (14) we obtain the following equations of motion

$$
\begin{aligned}
0 & =\square \alpha-e^{\frac{4}{3} \Phi-\frac{10}{3} \alpha}+n^{2} e^{\frac{4}{3} \Phi-\frac{16}{3} \alpha}, \\
0 & =\square \Phi+\frac{1}{2} n^{-2} e^{\frac{4}{3} \Phi-\frac{4}{3} \alpha}+\frac{1}{2} n^{2} e^{\frac{4}{3} \Phi-\frac{16}{3} \alpha}, \\
R_{5 \mu \nu} & =\frac{10}{3} \partial_{\mu} \alpha \partial_{\nu} \alpha+\frac{4}{3} \partial_{\mu} \Phi \partial_{\nu} \Phi-\frac{4}{3}\left(\partial_{\mu} \alpha \partial_{\nu} \Phi+\partial_{\nu} \alpha \partial_{\mu} \Phi\right)-\frac{1}{3} g_{\mu \nu},
\end{aligned}
$$


where we denote

$$
V \equiv n^{-2} e^{\frac{4}{3} \Phi-\frac{4}{3} \alpha}+2 e^{\frac{4}{3} \Phi-\frac{10}{3} \alpha}-n^{2} e^{\frac{4}{3} \Phi-\frac{16}{3} \alpha} .
$$

\section{Finite temperature MN geometry: analytic solutions and the equation of state}

We begin with deriving equations of motion describing finite temperature deformation of the chirally symmetric MN solution $[13,15]$. The regular horizon solutions of these equations are characterized by two independent parameters: the value of a dilaton and the $S_{2}$ (see (6) ) radius at the horizon. These parameters are related to the temperature and the strong coupling scale ${ }^{8}$ of the dual SYM theory (alternatively the Kaluza-Klein scale of the compactified LST). We analytically construct solutions for the nonextremal deformations perturbatively in inverse $S_{2}$ radius at the horizon, which corresponds to a high energy (near Hagedorn ) regime of the compactified LST [14]. We argue that keeping the strong coupling scale of the dual gauge theory fixed corresponds to a very specific dependence of the horizon value of a dilaton on an $S_{2}$ horizon radius ${ }^{9}$. We finally derive high-energy equation of state for the compactified LST and discuss the discrepancy with the previous proposals $[14,15]$.

\subsection{Background equations of motion for the finite temperature MN solu- tion}

Nonextremal deformations of the MN geometry are described by the following ansatz of the five-dimensional effective action (9)

$$
d s_{5}^{2}=n^{4 / 3} g^{\frac{2}{3}} e^{-\frac{4}{3} \Phi}\left(-f^{2} d t^{2}+d \bar{x}^{2}+\frac{1}{4} n^{2} d r^{2}\right),
$$

\footnotetext{
${ }^{8}$ The fact that only two parameters specify regular horizon solution implies that finite temperature gauge theory dual to nonextremal MN background indeed does not have a chemical potential, and thus falls in a class of systems discussed in section 1.

${ }^{9}$ This differs from the assumption in [14] where the high temperature thermodynamics of the MN solution was deduced assuming constant horizon value of the dilaton.
} 
where $f=f(r)$, also we have $\Phi=\Phi(r), \alpha \equiv \frac{1}{2} \ln g+\ln n=\frac{1}{2} \ln g(r)+\ln n$. In this case equations of motion (15) become

$$
\begin{aligned}
& 0=f^{\prime \prime}+[\ln g-2 \Phi]^{\prime} f^{\prime} \\
& 0=g^{\prime \prime}+[\ln f-2 \Phi]^{\prime} g^{\prime}+\frac{1-g}{2 g} \\
& 0=\Phi^{\prime \prime}+[\ln f g-2 \Phi]^{\prime} \Phi^{\prime}+\frac{1+g^{2}}{8 g^{2}}
\end{aligned}
$$

where prime denotes derivative with respect to $r$. Additionally we have a first order constraint

$$
0=[\ln g]^{\prime}\left[\ln g^{\frac{1}{8}} f^{\frac{1}{2}}-\Phi\right]^{\prime}+\Phi^{\prime}[\Phi-\ln f]^{\prime}+\frac{1-2 g-g^{2}}{16 g^{2}} .
$$

We specifically choose such a parametrization that all $n$-dependence drops out in (18), (19). In this parametrization the extremal chirally symmetric solution of MN [6] takes a very simple form

$$
f=1, \quad g=r, \quad \Phi=\phi_{0}-\frac{1}{4} r+\frac{1}{4} \ln r .
$$

Notice from (20) that MN asymptotics (as $r \rightarrow+\infty$ ) can be summarized in a coordinate independent way as

$$
\Phi=-\frac{1}{4} g+\mathcal{O}(\ln g) .
$$

We propose that enforcing the asymptotic (21) even off the extremality is equivalent to keeping the Kaluza-Klein scale of the compactified LST fixed. We will show later that this implies a particular dependence of the horizon value of the dilaton on the $S_{2}$ radius, also evaluated at the horizon.

Nonextremal solutions of (18) are best analyzed using a new radial gauge

$$
x \equiv f
$$

This gauge choice is nonsingular because $f(r)$ is a smooth, monotonic function ${ }^{10}$ of $r$ in (17). Furthermore, it reduces the number of independent functions describing the nonextremal MN background to two: $g(x), \Phi(x)$. With the radial coordinate as in (22), the black brane horizon is at $x=0$ and the boundary is at $x=1$. The background

\footnotetext{
${ }^{10}$ We explicitly verified this fact.
} 
equations of motion (18) take the form

$$
\begin{aligned}
& 0=g^{\prime \prime}-\frac{g^{2}+3 g-2}{g\left(g^{2}+2 g-1\right)}\left(g^{\prime}\right)^{2}+\frac{8 x(g-1) \Phi^{\prime}+g^{2}-2 g+3}{x\left(g^{2}+2 g-1\right)} g^{\prime}-\frac{8 \Phi^{\prime} g(g-1)\left(x \Phi^{\prime}-1\right)}{x\left(g^{2}+2 g-1\right)} \\
& 0=\Phi^{\prime \prime}+\frac{2\left(g^{2}+1\right)}{g^{2}+2 g-1}\left(\Phi^{\prime}\right)^{2}-\frac{2 x\left(g^{2}+1\right) g^{\prime}+g^{3}-2 g^{2}+3 g}{x g\left(g^{2}+2 g-1\right)} \Phi^{\prime}+\frac{\left(g^{2}+1\right) g^{\prime}\left(x g^{\prime}+4 g\right)}{4 x g^{2}\left(g^{2}+2 g-1\right)}
\end{aligned}
$$

where all derivatives are now with respect to $x$. Regularity at the horizon implies the following perturbative expansion as $x \rightarrow 0_{+}[13]$

$$
g=a_{0}^{2}+\sum_{n=1}^{\infty} a_{n} x^{2 n}, \quad \Phi=\phi_{0}+\sum_{n=1}^{\infty} p_{n} x^{2 n}
$$

where $n a_{0}$ and $\phi_{0}$ are the radius of the $S_{2}$ and the value of the dilaton at the horizon respectively. The first couple terms of the perturbative expansion take values

$$
\begin{aligned}
& g_{1}=\frac{4 a_{0}^{2}\left(1-a_{0}^{2}\right)}{a_{0}^{4}+1} p_{1}, \quad g_{2}=\frac{4 a_{0}^{2}\left(a_{0}^{4}+3 a_{0}^{2}-1\right)\left(a_{0}^{2}-1\right)}{\left(a_{0}^{4}+1\right)^{2}} p_{1}^{2}, \\
& p_{2}=-\frac{a_{0}^{8}+2 a_{0}^{6}+1}{\left(a_{0}^{4}+1\right)^{2}} p_{1}^{2} .
\end{aligned}
$$

Notice that $p_{1}$ is not fixed; in fact, it is easy to see that $a_{n} \propto p_{1}^{n}, p_{n} \propto p_{1}^{n}$. The latter is related to the exact scaling symmetry of (23): $x \rightarrow \lambda x$ with $g$, $\Phi$ kept invariant ${ }^{11}$. While $a_{0}$ and $\phi_{0}$ are independent physical parameters of the nonextremal solution (related to the temperature and the strong coupling scale of the gauge theory), $p_{1}$ is not. As explained in [13], for a fixed values of $\left\{a_{0}, \phi_{0}\right\}$, the parameter $p_{1}$ must be fixed in such a way that the boundary of the nonextremal geometry is indeed at $x=1$. This is equivalent to requiring that $g \rightarrow+\infty$ as $x \rightarrow 1_{-}$.

As shown in $[13,15]$ the global solution of (23) (with a regular horizon) is crucially sensitive to the value of $a_{0}$. For $a_{0}>1$ the solution is singularity-free in the bulk, and approach asymptotically the MN solution (21). For $a_{0}<1$ the solution has a naked singularity in the bulk. Finally, for $a_{0}=1$, the exact solution takes form

$$
g=1, \quad \Phi=\phi_{0}+\frac{1}{2} \ln \left(1-x^{2}\right) .
$$

Clearly, (26) does not asymptote to MN solution near the boundary. Quite interestingly, the $a_{0}=1$ nonextremal background has an exact string world-sheet description $[15]$.

\footnotetext{
${ }^{11}$ This scaling symmetry is broken by the boundary, located at $x=1$.
} 


\subsection{Analytic nonextremal solutions of eq. (23) for $a_{0} \gg 1$}

In previous section we derived equations of motion describing nonextremal deformations of the chirally symmetric MN background (23). The globally regular solutions are characterized by the horizon boundary condition $a_{0}>1$. Geometrically, $n a_{0}$ is the radius of the $S_{2}$ (see (6)) of the string frame ten-dimensional geometry. Whenever $a_{0}>1$, the string frame size of $S_{2}$ monotonically increases as one approaches the boundary, diverging at the boundary. This $S_{2}$ is a two-cycle of the resolved conifold, that is being wrapped by the NS5 branes. Intuitively, the larger the $a_{0}$, the more locally flat the NS5 branes are. Thus we expect that the $a_{0} \rightarrow+\infty$ limit of the nonextremal MN solution to describe the non-extremal type IIB string theory NS5 branes. The thermodynamics of this system is Hagedorn [23]. We are unable to solve analytically (23) for arbitrary values of $a_{0}$. On the other hand, it is possible to solve these equations perturbatively in $a_{0}^{-2}$. Such perturbative solution describes corrections to the Hagedorn thermodynamics of NS5 branes wrapped on a large two-cycle. It is this regime where we test the proof of the Gubser-Mitra conjecture presented in section 1.

For large values of $a_{0}$ we seek solution to (23) in the form

$$
\begin{gathered}
g=a_{0}^{2}+\sum_{n=0}^{\infty} \frac{\mathcal{G}_{n}(x)}{a_{0}^{2 n}}, \\
\Phi=\phi_{0}+\sum_{n=0}^{\infty} \frac{\mathcal{P}_{n}(x)}{a_{0}^{2 n}} .
\end{gathered}
$$

It is straightforward to substitute ansatz (27) into (23), and solve resulting ODE's iteratively in $n$. Given a horizon boundary condition

$$
\left.g\right|_{\text {horizon }}=a_{0}^{2},\left.\quad \Phi\right|_{\text {horizon }}=\phi_{0},
$$

and the MN asymptotic, $\left\{\mathcal{G}_{n}(x), \mathcal{P}_{n}(x)\right\}$ are uniquely determined. In what follows we will need an explicit solution up to order $n=2$. We find

$$
\begin{gathered}
\mathcal{P}_{0}=\frac{1}{2} \ln \left(1-x^{2}\right), \\
\mathcal{G}_{0}=-2 \ln \left(1-x^{2}\right), \\
\mathcal{P}_{1}=-\ln \left(1-x^{2}\right), \\
\mathcal{G}_{1}=2 \ln \left(1-x^{2}\right)+4 \operatorname{dil} \log \left(x^{2}\right)+8 \ln x \ln \left(1-x^{2}\right)-\frac{2}{3} \pi^{2},
\end{gathered}
$$




$$
\begin{aligned}
\mathcal{P}_{2}= & 2 \ln \left(1-x^{2}\right)-\ln ^{2}\left(1-x^{2}\right)-2 \operatorname{dilog}\left(1-x^{2}\right), \\
\mathcal{G}_{2}= & -6 \ln \left(1-x^{2}\right)-8 \ln x \ln ^{2}\left(1-x^{2}\right)-8 \ln \left(1-x^{2}\right) \operatorname{polylog}\left(2,1-x^{2}\right) \\
& +8 \operatorname{polylog}\left(3,1-x^{2}\right)+12 \operatorname{dil} \log \left(1-x^{2}\right)+2 \ln ^{2}\left(1-x^{2}\right)-8 \zeta(3) .
\end{aligned}
$$

From (27), (29)-(31) it is obvious that the limit $a_{0} \rightarrow+\infty$ does not commute with the limit $x \rightarrow 1_{-}$. Thus, a correct order of limits must be specified. In what follows we always assume that the $a_{0}$ limit is taken first. The motivation for such a choice comes from the fact that as $a_{0} \rightarrow+\infty$ the temperature of the nonextremal MN solution (see (34)) approaches flat NS5 brane Hagedorn temperature. Moreover, such a scaling implies that in the strict $a_{0} \rightarrow+\infty$ limit only $n=0$ terms of (27) must be kept. In this case

$$
\begin{aligned}
g & =a_{0}^{2}+\mathcal{G}_{0} \rightarrow+\infty \\
\Phi & =\phi_{0}+\mathcal{P}_{0}=\phi_{0}+\frac{1}{2} \ln \left(1-x^{2}\right)
\end{aligned}
$$

which is precisely the geometry of the nonextremal flat NS5 branes [23]. Finally, such a scaling produces sound wave dispersion relation (see section 4) in agreement with that of the nonextremal flat NS5 brane system [30]. Thus, it is natural to identify the proposed regime with the near-Hagedorn regime of NS5 branes wrapped on a large twocycle. It is conceivable that some other order of limits would produce a system with a different thermodynamic (and hydrodynamic) description; we were not able to find a coherent description other than what we present here. It would be very interesting to find such a description (if it exists).

We conclude with section by noting ${ }^{12}$ that in the prescribed order of limits the MN asymptotic (21) is indeed satisfied provided we identify

$$
\phi_{0}=\hat{\phi}_{0}-\frac{1}{4} a_{0}^{2}+\mathcal{O}\left(\ln a_{0}\right)
$$

where $\hat{\phi}_{0}$ is independent of $a_{0}$.

\subsection{Large $a_{0}$ thermodynamics of the finite temperature MN solution}

In this section we study the thermodynamics of the LST compactified on a large twocycle of the resolved conifold.

\footnotetext{
${ }^{12}$ This follows from (32).
} 
Given the metric ansatz (17), and the regular horizon boundary conditions (24), we find the Hawking temperature of the black brane solution by identifying its inverse with the periodicity of the Euclidean time direction

$$
T=\frac{1}{4 \pi n}\left(-\frac{1}{2 p_{1}}\left(1+a_{0}^{-4}\right)\right)^{1 / 2} .
$$

Next we compute the Bekenstein-Hawking entropy density of the geometry (17). We find the three-dimensional area of the horizon $\mathcal{A}_{3}$ of the black brane to be

$$
\mathcal{A}_{3}=n^{2} a_{0}^{2} e^{-2 \phi_{0}} V_{3}
$$

where $V_{3}$ is the three-dimensional volume. The entropy density of the black brane is

$$
S=\frac{\mathcal{A}_{3}}{4 V_{3} G_{5}} \propto n^{5} a_{0}^{2} e^{-2 \phi_{0}}
$$

In the limit $a_{0} \gg 1$ we can use perturbative solution (27), (29)-(31) to determine

$$
p_{1}=-\frac{1}{2}+a_{0}^{-2}+\mathcal{O}\left(a_{0}^{-4}\right)
$$

From (34), (37) we see that in the limit $a_{0} \rightarrow+\infty[14]$

$$
T=T_{H}\left(1+a_{0}^{-2}+\mathcal{O}\left(a_{0}^{-4}\right)\right)
$$

where $T_{H}=1 /(4 \pi n)$ is the Hagedorn temperature of the six-dimensional LST.

To determine equation of state we need to compute the energy density (and the pressure) of the gravitational background (17). The correct way to do this is to study holographic renormalization [24-28] of the MN solution, much like equivalent problem recently solved for the cascading gauge theories [21]. In all cases we are aware of, the properly implemented holographic renormalization in the context of gauge theory/ string theory correspondence produces the entropy density and the pressure automatically satisfying the standard thermodynamic relations ${ }^{13}$

$$
P=-\mathcal{F}, \quad d \mathcal{E}=T d S
$$

Lacking the holographic renormalization for the MN background, one could still deduce the remaining thermodynamic quantities (besides the entropy density) by enforcing (39). The subtlety of the latter prescription in the context of MN geometry is that

\footnotetext{
${ }^{13}$ For asymptotically locally $A d S$ backgrounds this was rigorously proven in [29].
} 
a priori, one does not know whether $\phi_{0}$ depends on $a_{0}$ once the physical properties of the gauge theory (in our case the strong coupling scale) is kept fixed, and if so, what is the precise relation. Identical problem arises in the study of thermodynamics of the cascading gauge theories [18-21]. There, the entropy density also depends on two parameters: the five-form flux at the black brane horizon, and the nonextremality parameter (temperature). Moreover, for the correct thermodynamics, the five-form flux at the horizon must depend on the temperature. This was explicitly demonstrated in [21]. The correct horizon flux/temperature relation for the cascading gauge theories was determined without holographic renormalization in $[20]^{14}$ by requiring that the glueball mass scale of the theory is kept fixed. The same conclusion could be reached ${ }^{15}$ by keeping the 'holographic scale' (the radial coordinate) 'fixed' - independent of the nonextremality parameter. One way to achieve this is to require that the asymptotic relation between two supergravity modes (which are affected by the temperature only at the subleading order near the boundary) are kept fixed - the same as in the extremal (supersymmetric) case. In the case of nonextremal MN deformation, this would be the statement that the asymptotic relation between the $S_{2}$ radius and the dilaton near the boundary of (6) is the same as in the extremal MN solution. For large $a_{0}$, this leads to (33). In our analysis of the MN thermodynamics we assume (33) to be correct. Then, using (36), (38), (39) we find

$$
\begin{aligned}
v_{s}^{2} & =\frac{S}{c_{V}}=S \frac{\partial T}{\partial \mathcal{E}}=\frac{S}{T} \frac{\partial T}{\partial S}=\frac{\partial \ln T}{\partial \ln S}=\frac{\partial \ln T / \partial a_{0}}{\partial \ln S / \partial a_{0}} \\
& =-\frac{2}{a_{0}^{4}}+\mathcal{O}\left(a_{0}^{-6}\right) .
\end{aligned}
$$

Notice that for $a_{0} \gg 1, v_{s}$ is purely imaginary; moreover it vanishes in the limit $a_{0} \rightarrow+\infty$, in agreement with the computation for the speed of sound in six dimensional LST [30]. In the next section we reproduce (40) directly from the computation of the lowest quasinormal mode in finite temperature MN geometry.

We conclude this section by commenting on the previous proposals for the equations of state for the finite temperature MN solution.

- In [14] we discussed the thermodynamics of MN solution assuming that

$$
\frac{\partial \phi_{0}}{\partial a_{0}}=0
$$

\footnotetext{
${ }^{14}$ The authors then computed the remaining thermodynamic quantities from (39).

${ }^{15}$ I would like to thank Ofer Aharony for discussing this point.
} 
We argued here that (41) is incorrect, rather one should use (33). The speed of sound deduced under the assumption (41) disagrees with the explicit computation of the lowest quasinormal mode dispersion relation of the next section.

- In [15] the authors computed the free energy of the finite temperature MN geometry using a reference background subtraction prescription [31]. It is known that background subtraction as a method for computing the free energy does not work for charged black holes in $A d S_{5}$, and for the supergravity dual to mass deformed $\mathcal{N}=4 \mathrm{SYM}$ theory [32-34]. We claim here that is does not work for the nonextremal MN background as well. The conclusion of the background subtraction method to finite temperature MN geometry was (see Figs. 20,21 of [15]) that in the limit $S \rightarrow+\infty$ (the limit of standard flat NS5 brane thermodynamics) both $\mathcal{E} \propto S$ and $-P=\mathcal{F} \propto-S$. Thus in the high energy (entropy) limit $\mathcal{E} \propto P$. So in the limit $S \rightarrow+\infty$

$$
v_{s}^{2}=\frac{\partial \mathcal{P}}{\partial \mathcal{E}} \rightarrow \text { constant } \neq 0
$$

which contradicts the flat LST result [30].

\section{Sound wave in nonextremal MN geometry}

The general prescription for the evaluation of the quasinormal spectra of the nonextremal gravitational backgrounds was explained in [8]. The application of the procedure to a particular solution is very straightforward. The discussion here closely resembles [22], so we outline only the main steps.

Consider fluctuations in the background geometry (17)

$$
\begin{aligned}
g_{\mu \nu} & \rightarrow g_{\mu \nu}^{b}+h_{\mu \nu}, \\
g & \rightarrow g^{b}+g_{1}, \\
\Phi & \rightarrow \Phi^{b}+\Phi_{1},
\end{aligned}
$$

where $\left\{g_{\mu \nu}^{b}, g^{b}, \Phi^{b}\right\}$ are the black brane background configuration (satisfying (18), (19)), and $\left\{h_{\mu \nu}, g_{1}, \Phi_{1}\right\}$ are the fluctuations. We choose the gauge

$$
h_{t r}=h_{x_{i} r}=h_{r r}=0 .
$$

Additionally, we assume that all the fluctuations depend only on $\left(t, x_{3}, r\right)$, i.e., we have an $O(2)$ rotational symmetry in the $x_{1} x_{2}$ plane. At a linearized level we find that the 
following sets of fluctuations to decouple from each other

$$
\begin{aligned}
& \left\{h_{x_{1} x_{2}}\right\}, \\
& \left\{h_{x_{1} x_{1}}-h_{x_{2} x_{2}}\right\}, \\
& \left\{h_{t x_{1}}, h_{x_{1} x_{3}}\right\}, \\
& \left\{h_{t x_{2}}, h_{x_{2} x_{3}}\right\}, \\
& \left\{h_{t t}, h_{a a} \equiv h_{x_{1} x_{1}}+h_{x_{2} x_{2}}, h_{t x_{3}}, h_{x_{3} x_{3}}, g_{1}, \Phi_{1}\right\} .
\end{aligned}
$$

The last set of fluctuations is a holographic dual to the sound waves in MN model which is of interest here. Introduce

$$
\begin{aligned}
h_{t t} & ==e^{-i \omega t+i q x_{3}}\left[n^{4 / 3}\left(g^{b}\right)^{\frac{2}{3}} e^{-\frac{4}{3} \Phi^{b}} f_{b}^{2}\right] H_{t t}, \\
h_{t z} & ==e^{-i \omega t+i q x_{3}}\left[n^{4 / 3}\left(g^{b}\right)^{\frac{2}{3}} e^{-\frac{4}{3} \Phi^{b}}\right] H_{t z}, \\
h_{a a} & ==e^{-i \omega t+i q x_{3}}\left[n^{4 / 3}\left(g^{b}\right)^{\frac{2}{3}} e^{-\frac{4}{3} \Phi^{b}}\right] H_{a a}, \\
h_{z z} & ==e^{-i \omega t+i q x_{3}}\left[n^{4 / 3}\left(g^{b}\right)^{\frac{2}{3}} e^{-\frac{4}{3} \Phi^{b}}\right] H_{z z}, \\
g_{1} & =e^{-i \omega t+i q x_{3}} \phi \\
\Phi_{1} & =e^{-i \omega t+i q x_{3}} \psi,
\end{aligned}
$$

where $\left\{H_{t t}, H_{t z}, H_{a a}, H_{z z}, \phi, \psi\right\}$ are functions of a radial coordinate only. Further we introduce diffeomorphism invariant fluctuations

$$
\begin{aligned}
Z_{H} & =4 \frac{q}{\omega} H_{t z}+2 H_{z z}-H_{a a}\left(1-\frac{q^{2}}{\omega^{2}} f_{b} \frac{2 f_{b} g_{b} \Phi_{b}^{\prime}-f_{b} g_{b}^{\prime}-3 g_{b} f_{b}^{\prime}}{2 g_{b} \Phi_{b}^{\prime}-g_{b}^{\prime}}\right)+2 \frac{q^{2}}{\omega^{2}} f_{b}^{2} H_{t t}, \\
Z_{g} & =\phi-\frac{3 g_{b} g_{b}^{\prime}}{4 g_{b}^{\prime}-8 g_{b} \Phi_{b}^{\prime}} H_{a a}, \\
Z_{\Phi} & =\psi-\frac{3 g_{b} \Phi_{b}^{\prime}}{4 g_{b}^{\prime}-8 g_{b} \Phi_{b}^{\prime}} H_{a a} .
\end{aligned}
$$

The quasinormal mode spectrum is determined [8] by imposing the incoming boundary condition at the horizon, and the Dirichlet condition at the boundary on the diffeomorphism invariant fluctuations (46). So at the horizon $\left(f_{b} \rightarrow 0_{+}\right)$we have (see also [35,22])

$$
Z_{H}(r)=f_{b}^{-\frac{i \omega}{2 \pi T}} z_{H}(r), \quad Z_{g}(r)=f_{b}^{-\frac{i \omega}{2 \pi T}} z_{g}(r), \quad Z_{\Phi}(r)=f_{b}^{-\frac{i \omega}{2 \pi T}} z_{\Phi}(r)
$$


where $\left\{z_{H}, z_{g}, z_{\Phi}\right\}$ are regular at the horizon; while at the boundary $\left(f_{b} \rightarrow 1_{-}\right)$

$$
z_{H} \rightarrow 0, \quad z_{g} \rightarrow 0, \quad z_{\Phi} \rightarrow 0 .
$$

Taking the hydrodynamic limit,

$$
\omega \rightarrow 0, \quad q \rightarrow 0, \quad \frac{\omega}{q} \rightarrow v_{s},
$$

we find the following set of equations (in the radial coordinate (22))

$$
\begin{aligned}
& 0=z_{H}^{\prime \prime}+A_{H} z_{H}^{\prime}+B_{H} z_{H}+C_{H} z_{g}+D_{H} z_{\Phi}, \\
& 0=z_{g}^{\prime \prime}+A_{g} z_{g}^{\prime}+B_{g} z_{g}+C_{g} z_{\Phi}+D_{g}\left(x z_{H}^{\prime}-z_{H}\right), \\
& 0=z_{\Phi}^{\prime \prime}+A_{\Phi} z_{\Phi}^{\prime}+B_{\Phi} z_{\Phi}+C_{\Phi} z_{g}+D_{\Phi}\left(x z_{H}^{\prime}-z_{H}\right),
\end{aligned}
$$

where the coefficients are given explicitly in the Appendix. The symmetry of these coefficients imply that the equation for

$$
\lambda \equiv z_{g}-z_{\Phi}
$$

is particularly simple ${ }^{16}$ :

$$
0=x \lambda^{\prime \prime}+\lambda^{\prime} .
$$

A unique solution of (52) consistent with boundary conditions (477), (48) is $\lambda(x) \equiv 0$. Thus we conclude that in the hydrodynamic approximation

$$
z_{g}=z_{\Phi} .
$$

We now proceed with solving (50), subject to the boundary conditions (47), (48) in the approximation $a_{0} \gg 1$. Using results of section 3.2 and (53), we find

$$
\begin{aligned}
0= & z_{H}^{\prime \prime}+\left\{\frac{1-3 x^{2}-2 v_{s}^{2}}{x\left(-2 v_{s}^{2}+1+x^{2}\right)}-\frac{16 x\left(1-v_{s}^{2}\right)}{\left.a_{0}^{4}\left(-2 v_{s}^{2}+1+x^{2}\right)^{2}\right)}+\mathcal{O}\left(a_{0}^{-6}\right)\right\} z_{H}^{\prime} \\
& +\left\{\frac{32\left(1-2 v_{s}^{2}\right)}{a_{0}^{2}\left(-2 v_{s}^{2}+1+x^{2}\right) v_{s}^{2}}+\frac{32\left(3+2\left(1-x^{2}\right) \ln \left(1-x^{2}\right)-6 v_{s}^{2}+x^{2}\left(1+2 v_{s}^{2}\right)\right)}{a_{0}^{4}\left(-1+x^{2}\right)\left(-2 v_{s}^{2}+1+x^{2}\right) v_{s}^{2}}\right. \\
& \left.+\mathcal{O}\left(a_{0}^{-6}\right)\right\} z_{g}+\left\{\frac{16\left(1-v_{s}^{2}\right)}{-2 v_{s}^{2}+1+x^{2}}+\frac{16 x^{2}}{a_{0}^{4}\left(-2 v_{s}^{2}+1+x^{2}\right.}+\mathcal{O}\left(a_{0}^{-6}\right)\right\} z_{H} \\
& 0=z_{g}^{\prime \prime}+\frac{1}{x} z_{g}^{\prime}+\left\{\frac{8}{a_{0}^{2}\left(1-x^{2}\right)^{2}}+\frac{16\left(\ln \left(1-x^{2}\right)-3\right)}{\left(1-x^{2}\right)^{2} a_{0}^{4}}+\mathcal{O}\left(a_{0}^{-6}\right)\right\} z_{g} \\
& -\frac{4 v_{s}^{2}\left(x z_{H}^{\prime}-z_{H}\right)\left(x^{2}+\ln \left(1-x^{2}\right)\left(1-x^{2}\right)\right)}{a_{0}^{4} x^{4}\left(-2 v_{s}^{2}+1+x^{2}\right)\left(1-x^{2}\right)}\left\{1+\mathcal{O}\left(a_{0}^{-2}\right)\right\} .
\end{aligned}
$$

Notice that eqs. (54), (55) are perturbative in $a_{0}^{-2}$, but exact in $v_{s}$.

\footnotetext{
${ }^{16}$ This is true only in the hydrodynamic approximation (49).
} 


\subsection{Leading order solution of eqs. (54), (55)}

To leading order in $a_{0}^{-2}$ we have from (55)

$$
0=z_{g}^{\prime \prime}+\frac{1}{x} z_{g}^{\prime}
$$

which subject to the boundary conditions (47), (48) has a unique solution

$$
z_{g}^{\text {leading }}=0 .
$$

To leading order in $a_{0}^{-2}$ we have from (154)

$$
0=z_{H}^{\prime \prime}+\frac{1-3 x^{2}-2 v_{s}^{2}}{x\left(-2 v_{s}^{2}+1+x^{2}\right)} z_{H}^{\prime}+\frac{4}{-2 v_{s}^{2}+1+x^{2}} z_{H} .
$$

The general solution of (58) takes the form

$$
z_{H}=C_{1}\left\{1-x^{2}-2 v_{s}^{2}\right\}+C_{2}\left\{\left(1-x^{2}-2 v_{s}^{2}\right) \ln x+2-4 v_{s}^{2}\right\},
$$

where $C_{i}$ are the two integration constants. The incoming wave boundary condition (47) implies that $C_{2}=0$. The Dirichlet condition at the boundary then determines

$$
\left(v_{s}^{\text {leading }}\right)^{2}=0
$$

which is consistent with results of [30]. Without loss of generality we can set $C_{1}=1$, thus

$$
z_{H}^{\text {leading }}=1-x^{2}
$$

\subsection{Next-to-leading order solution of eqs. (54), (55)}

The next-to-leading corrections to $\left\{z_{g}^{\text {leading }}, z_{H}^{\text {leading }}\right\}$ must satisfy the Dirichlet condition both at the horizon and the boundary.

Analyzing (55) it is easy to see that next-to-leading correction to $z_{g}^{\text {leading }}, z_{g}^{\text {ntl }} \propto$ $\frac{\left(v_{s}^{n t l}\right)^{2}}{a_{0}^{4}}$. It satisfies the following equation

$$
0=\left[z_{g}^{n t l}\right]^{\prime \prime}+\frac{1}{x}\left[z_{g}^{n t l}\right]^{\prime}+\frac{4\left(v_{s}^{n t l}\right)^{2}\left(x^{2}+\ln \left(1-x^{2}\right)\left(1-x^{2}\right)\right)}{a_{0}^{4} x^{4}\left(1-x^{2}\right)},
$$

where we substituted the leading solution for $z_{H}$, (61), and used the fact that the speed of sound vanishes to leading order in $a_{0}^{-2}$, (60). A unique solution to (62) satisfying all boundary conditions takes the form

$$
z_{g}^{n t l}=-\frac{\left(v_{s}^{n t l}\right)^{2}}{a_{0}^{4}} \frac{\left(1-x^{2}\right) \ln \left(1-x^{2}\right)}{x^{2}} .
$$


Next, consider next-to-leading correction to $z_{H}^{\text {leading }}$. Again, given the leading order solutions and (63), it is clear from (154) that $z_{H}^{n t l} \propto a_{0}^{-4},\left(v_{s}^{n t l}\right)^{2} \propto a_{0}^{-4}$. Setting

$$
\left(v_{s}^{n t l}\right)^{2}=\frac{\beta}{a_{0}^{4}},
$$

we find from (154)

$$
0=\left[z_{H}^{n t l}\right]^{\prime \prime}+\frac{1-3 x^{2}}{x\left(1+x^{2}\right)}\left[z_{H}^{n t l}\right]^{\prime}+\frac{4}{1+x^{2}} z_{H}^{n t l}+\frac{16+8 \beta}{1+x^{2}} .
$$

The general solution to (65) takes form

$$
z_{H}^{n t l}=C_{3}\left\{1-x^{2}\right\}+C_{4}\left\{\left(1-x^{2}\right) \ln x+2\right\}-4-2 \beta,
$$

where $C_{i}$ are the two integration constants. Regularity of the solution at the horizon implies that $C_{4}=0$; the Dirichlet condition at the boundary implies $\beta=-2$; finally, the horizon boundary condition on the next-to-leading correction implies $C_{3}=0$. Thus we conclude ${ }^{17}$

$$
z_{H}^{n t l}=\mathcal{O}\left(a_{0}^{-6}\right)
$$

and

$$
v_{s}^{2}=-\frac{2}{a_{0}^{4}}+\mathcal{O}\left(a_{0}^{-6}\right),
$$

in precise agreement with (40).

\section{Conclusion}

In this paper we presented a simple proof of the Gubser-Mitra conjecture [3] for the supergravity backgrounds with translationary invariant horizons what can be interpreted as holographic duals to strongly coupled finite temperature gauge theories. We illustrated the discussion with the study of thermodynamics and the hydrodynamics of the MN [6] geometries. We explicitly demonstrated that finite temperature MN background in the near Hagedorn regime has a negative specific heat, and the tachyonic lowest quasinormal mode. Moreover, the specific heat and the speed of sound (extracted from the dispersion relation for the lowest quasinormal mode) satisfy (5), which is predicted by our proof of the Gubser-Mitra conjecture.

\footnotetext{
${ }^{17}$ In order to evaluate $z_{H}^{n t l}$ explicitly, one needs to know $n=3$ functional coefficients of (27). This is not necessary for the evaluation of the speed of sound to first nonvanishing order.
} 
Since small fluctuations of a thermodynamic system are described by hydrodynamics, it is an interesting question as to whether there are other instabilities (besides the sound mode) in the thermodynamic system with a negative specific heat ${ }^{18}$. We do not know the answer in general, however, in the specific example of the MN model, we believe that there are none. First, the shear viscosity of the MN system is universally [36-39] $S /(4 \pi)$; thus, it is positive. Second, both the bulk viscosity and the R-charge diffusion constant of the LST [30] is finite, and positive. Our analysis suggests that the $a_{0} \rightarrow+\infty$ limit of the MN model smoothly reproduces six-dimensional LST results, thus we find it unlikely that the bulk viscosity or the R-charge diffusion constant would be negative for large $a_{0}$.

\section{Acknowledgments}

I would like to thank Ofer Aharony, Pavel Kovtun and Andrei Starinets for valuable discussions. Research at Perimeter Institute is supported in part by funds from NSERC of Canada. I gratefully acknowledge support by NSERC Discovery grant. I would like to thank Aspen Center for Physics for hospitality where this work was completed.

\section{Appendix}

Coefficients of fluctuation equations (50):

$$
\begin{gathered}
A_{H}=\frac{x\left(4 x g_{b}^{\prime} \Phi_{b}^{\prime} g_{b}+g_{b} g_{b}^{\prime}-2 x g_{b}^{\prime 2}-2 \Phi_{b}^{\prime} g_{b}^{2}-4 x \Phi_{b}^{\prime 2} g_{b}^{2}\right)-v_{s}^{2}\left(-g_{b}^{\prime}+2 \Phi_{b}^{\prime} g_{b}\right)^{2}}{\left(x\left(2 x \Phi_{b}^{\prime} g_{b}-g_{b}-x g_{b}^{\prime}\right)-v_{s}^{2}\left(-g_{b}^{\prime}+2 \Phi_{b}^{\prime} g_{b}\right)\right) x\left(-g_{b}^{\prime}+2 \Phi_{b}^{\prime} g_{b}\right)}, \\
B_{H}=\frac{-8 \Phi_{b}^{\prime} g_{b}^{\prime} g_{b}+8 \Phi_{b}^{\prime 2} g_{b}^{2}+3 g_{b}^{\prime 2}}{\left(-g_{b}^{\prime}+2 \Phi_{b}^{\prime} g_{b}\right)\left(x\left(2 x \Phi_{b}^{\prime} g_{b}-g_{b}-x g_{b}^{\prime}\right)-v_{s}^{2}\left(-g_{b}^{\prime}+2 \Phi_{b}^{\prime} g_{b}\right)\right)},
\end{gathered}
$$

\footnotetext{
${ }^{18} \mathrm{I}$ would like to thank Andrei Starinets for discussing this.
} 


$$
\begin{aligned}
C_{H}= & \frac{1}{3 g_{b}\left(-g_{b}^{\prime}+2 \Phi_{b}^{\prime} g_{b}\right)\left(2 x^{2} \Phi_{b}^{\prime} g_{b}-x g_{b}-x^{2} g_{b}^{\prime}+v_{s}^{2}\left(g_{b}^{\prime}-2 \Phi_{b}^{\prime} g_{b}\right)\right)\left(-1+g_{b}^{2}+2 g_{b}\right) v_{s}^{2}} \\
& \times\left(4 x ( - 3 x g _ { b } ^ { \prime } g _ { b } ^ { 2 } - 3 x g _ { b } ^ { \prime } + 1 2 x \Phi _ { b } ^ { \prime } g _ { b } - 1 2 x \Phi _ { b } ^ { \prime } g _ { b } ^ { 2 } - 8 g _ { b } + 2 g _ { b } ^ { 3 } + 1 0 g _ { b } ^ { 2 } ) \left(-8 \Phi_{b}^{\prime} g_{b}^{2}\right.\right. \\
& \left.+4 g_{b} g_{b}^{\prime}+8 x \Phi_{b}^{\prime 2} g_{b}^{2}-8 x g_{b}^{\prime} \Phi_{b}^{\prime} g_{b}+x g_{b}^{\prime 2}\right)+v_{s}^{2}\left(-32 g_{b}^{\prime 2} g_{b}^{3}+512 \Phi_{b}^{\prime 2} g_{b}^{3}+96 g_{b}^{4} x \Phi_{b}^{\prime 2} g_{b}^{\prime}\right. \\
& -96 x g_{b}^{\prime 2} \Phi_{b}^{\prime} g_{b}^{3}+480 x \Phi_{b}^{\prime 2} g_{b}^{\prime} g_{b}^{2}+48 x g_{b}^{\prime 2} \Phi_{b}^{\prime} g_{b}^{2}-144 x g_{b}^{\prime 2} \Phi_{b}^{\prime} g_{b}-384 x \Phi_{b}^{\prime 2} g_{b}^{\prime} g_{b}^{3} \\
& +128 g_{b}^{4} \Phi_{b}^{\prime} g_{b}^{\prime}+384 g_{b}^{4} x \Phi_{b}^{\prime 3}-512 \Phi_{b}^{\prime} g_{b}^{\prime} g_{b}^{2}+640 \Phi_{b}^{\prime} g_{b}^{\prime} g_{b}^{3}-384 x \Phi_{b}^{\prime 3} g_{b}^{3}+12 x g_{b}^{\prime 3} g_{b}^{2} \\
& \left.\left.-128 g_{b}^{5} \Phi_{b}^{\prime 2}-640 g_{b}^{4} \Phi_{b}^{\prime 2}+12 x g_{b}^{\prime 3}+128 g_{b}^{\prime 2} g_{b}-160 g_{b}^{\prime 2} g_{b}^{2}\right)\right)
\end{aligned}
$$

$$
\begin{gathered}
D_{H}=\frac{8 x\left(8 \Phi_{b}^{\prime} g_{b}^{2}-4 g_{b} g_{b}^{\prime}-8 x \Phi_{b}^{\prime 2} g_{b}^{2}+8 x g_{b}^{\prime} \Phi_{b}^{\prime} g_{b}-x g_{b}^{\prime 2}\right)+32\left(-g_{b}^{\prime}+2 \Phi_{b}^{\prime} g_{b}\right)^{2} v_{s}^{2}}{3\left(-g_{b}^{\prime}+2 \Phi_{b}^{\prime} g_{b}\right)\left(2 x^{2} \Phi_{b}^{\prime} g_{b}-x g_{b}-x^{2} g_{b}^{\prime}+v_{s}^{2}\left(g_{b}^{\prime}-2 \Phi_{b}^{\prime} g_{b}\right)\right) v_{s}^{2}} \\
A_{g}=A_{\Phi}=\frac{1}{x}
\end{gathered}
$$

$$
\begin{aligned}
B_{g}= & C_{\Phi}=\frac{-8 \Phi_{b}^{\prime} g_{b}^{2}+4 g_{b} g_{b}^{\prime}+8 x \Phi_{b}^{\prime 2} g_{b}^{2}-8 x g_{b}^{\prime} \Phi_{b}^{\prime} g_{b}+x g_{b}^{\prime 2}}{6 x g_{b}^{2}\left(-1+g_{b}^{2}+2 g_{b}\right)\left(2 x^{2} \Phi_{b}^{\prime} g_{b}-x g_{b}-x^{2} g_{b}^{\prime}+v_{s}^{2}\left(g_{b}^{\prime}-2 \Phi_{b}^{\prime} g_{b}\right)\right)\left(-g_{b}^{\prime}+2 \Phi_{b}^{\prime} g_{b}\right)} \\
& \times\left(x \left(24 x \Phi_{b}^{\prime 2} g_{b}^{3}-48 x \Phi_{b}^{\prime 2} g_{b}^{2}+24 x g_{b}^{\prime} \Phi_{b}^{\prime} g_{b}+32 \Phi_{b}^{\prime} g_{b}^{2}-20 \Phi_{b}^{\prime} g_{b}^{3}+3 x g_{b}^{\prime 2} g_{b}^{2}-3 x g_{b}^{\prime 2}\right.\right. \\
& \left.\left.-8 g_{b} g_{b}^{\prime}-2 g_{b}^{\prime} g_{b}^{3}\right)+\left(48 \Phi_{b}^{\prime 2} g_{b}^{2}-24 \Phi_{b}^{\prime 2} g_{b}^{3}-24 \Phi_{b}^{\prime} g_{b}^{\prime} g_{b}+3 g_{b}^{\prime 2}-3 g_{b}^{\prime 2} g_{b}^{2}\right) v_{s}^{2}\right)
\end{aligned}
$$

$$
C_{g}=B_{\Phi}=\frac{\left(-8 \Phi_{b}^{\prime} g_{b}^{2}+4 g_{b} g_{b}^{\prime}+8 x \Phi_{b}^{\prime 2} g_{b}^{2}-8 x g_{b}^{\prime} \Phi_{b}^{\prime} g_{b}+x g_{b}^{\prime 2}\right)\left(4 \Phi_{b}^{\prime} g_{b}^{2}-4 \Phi_{b}^{\prime} g_{b}+g_{b}^{2} g_{b}^{\prime}+g_{b}^{\prime}\right)}{3 g_{b}\left(-g_{b}^{\prime}+2 \Phi_{b}^{\prime} g_{b}\right)\left(2 x^{2} \Phi_{b}^{\prime} g_{b}-x g_{b}-x^{2} g_{b}^{\prime}+v_{s}^{2}\left(g_{b}^{\prime}-2 \Phi_{b}^{\prime} g_{b}\right)\right)\left(-1+g_{b}^{2}+2 g_{b}\right)}
$$

$$
\begin{aligned}
D_{g}= & D_{\Phi}= \\
& \frac{\left(8 \Phi_{b}^{\prime} g_{b}^{2}-4 g_{b} g_{b}^{\prime}-8 x \Phi_{b}^{\prime 2} g_{b}^{2}+8 x g_{b}^{\prime} \Phi_{b}^{\prime} g_{b}-x g_{b}^{\prime 2}\right)\left(4 \Phi_{b}^{\prime} g_{b}^{2}-4 \Phi_{b}^{\prime} g_{b}+g_{b}^{2} g_{b}^{\prime}+g_{b}^{\prime}\right) v_{s}^{2}}{8 x^{2} g_{b}\left(-1+g_{b}^{2}+2 g_{b}\right)\left(2 x^{2} \Phi_{b}^{\prime} g_{b}-x g_{b}-x^{2} g_{b}^{\prime}+v_{s}^{2}\left(g_{b}^{\prime}-2 \Phi_{b}^{\prime} g_{b}\right)\right)\left(-g_{b}^{\prime}+2 \Phi_{b}^{\prime} g_{b}\right)}
\end{aligned}
$$




\section{References}

[1] J. M. Maldacena, "The large N limit of superconformal field theories and supergravity," Adv. Theor. Math. Phys. 2, 231 (1998) [Int. J. Theor. Phys. 38, 1113 (1999)] arXiv:hep-th/9711200.

[2] O. Aharony, S. S. Gubser, J. M. Maldacena, H. Ooguri and Y. Oz, "Large N field theories, string theory and gravity," Phys. Rept. 323, 183 (2000) arXiv:hep-th/9905111.

[3] S. S. Gubser and I. Mitra, "Instability of charged black holes in anti-de Sitter space," arXiv:hep-th/0009126.

[4] H. S. Reall, "Classical and thermodynamic stability of black branes," Phys. Rev. D 64, 044005 (2001) arXiv:hep-th/0104071.

[5] S. S. Gubser and A. Ozakin, "Universality classes for horizon instabilities," JHEP 0305, 010 (2003) arXiv:hep-th/0301002.

[6] J. M. Maldacena and C. Nunez, "Towards the large N limit of pure $\mathrm{N}=1$ super Yang Mills," Phys. Rev. Lett. 86, 588 (2001) arXiv:hep-th/0008001.

[7] O. Aharony, S. Minwalla and T. Wiseman, "Plasma-balls in large N gauge theories and localized black holes," arXiv:hep-th/0507219.

[8] P. K. Kovtun and A. O. Starinets, "Quasinormal modes and holography," arXiv:hep-th/0506184.

[9] M. Berkooz, M. Rozali and N. Seiberg, "Matrix description of M theory on T**4 and T**5," Phys. Lett. B 408, 105 (1997) arXiv:hep-th/9704089.

[10] N. Seiberg, "New theories in six dimensions and matrix description of M-theory on $\mathrm{T}^{* *} 5$ and T**5/Z(2)," Phys. Lett. B 408, 98 (1997) arXiv:hep-th/9705221.

[11] A. H. Chamseddine and M. S. Volkov, "Non-Abelian BPS monopoles in N $=4$ gauged supergravity," Phys. Rev. Lett. 79, 3343 (1997) arXiv:hep-th/9707176.

[12] A. H. Chamseddine and M. S. Volkov, "Non-Abelian solitons in N $=4$ gauged supergravity and leading order string theory," Phys. Rev. D 57, 6242 (1998) arXiv:hep-th/9711181. 
[13] A. Buchel and A. R. Frey, "Comments on supergravity dual of pure $\mathrm{N}=1$ super Yang Mills theory with unbroken chiral symmetry," Phys. Rev. D 64, 064007 (2001) arXiv:hep-th/0103022.

[14] A. Buchel, "On the thermodynamic instability of LST," arXiv:hep-th/0107102.

[15] S. S. Gubser, A. A. Tseytlin and M. S. Volkov, "Non-Abelian 4-d black holes, wrapped 5-branes, and their dual descriptions," JHEP 0109, 017 (2001) arXiv:hep-th/0108205.

[16] I. R. Klebanov and A. A. Tseytlin, "Gravity duals of supersymmetric SU(N) x SU(N+M) gauge theories," Nucl. Phys. B 578, 123 (2000) arXiv:hep-th/0002159.

[17] I. R. Klebanov and M. J. Strassler, "Supergravity and a confining gauge theory: Duality cascades and chiSB-resolution of naked singularities," JHEP 0008, 052 (2000) arXiv:hep-th/0007191.

[18] A. Buchel, "Finite temperature resolution of the Klebanov-Tseytlin singularity," Nucl. Phys. B 600, 219 (2001) arXiv:hep-th/0011146.

[19] A. Buchel, C. P. Herzog, I. R. Klebanov, L. A. Pando Zayas and A. A. Tseytlin, "Non-extremal gravity duals for fractional D3-branes on the conifold," JHEP 0104, 033 (2001) arXiv:hep-th/0102105.

[20] S. S. Gubser, C. P. Herzog, I. R. Klebanov and A. A. Tseytlin, "Restoration of chiral symmetry: A supergravity perspective," JHEP 0105, 028 (2001) arXiv:hep-th/0102172.

[21] O. Aharony, A. Buchel and A. Yarom, "Holographic renormalization of cascading gauge theories," Phys. Rev. D 72, 066003 (2005) arXiv:hep-th/0506002.

[22] P. Benincasa, A. Buchel and A. O. Starinets, "Sound waves in strongly coupled non-conformal gauge theory plasma," arXiv:hep-th/0507026

[23] J. M. Maldacena and A. Strominger, "Semiclassical decay of near-extremal fivebranes," JHEP 9712, 008 (1997) arXiv:hep-th/9710014.

[24] M. Henningson and K. Skenderis, "The holographic Weyl anomaly," JHEP 9807, 023 (1998) arXiv:hep-th/9806087. 
[25] V. Balasubramanian and P. Kraus, "A stress tensor for anti-de Sitter gravity," Commun. Math. Phys. 208, 413 (1999) arXiv:hep-th/9902121.

[26] S. de Haro, S. N. Solodukhin and K. Skenderis, "Holographic reconstruction of spacetime and renormalization in the AdS/CFT correspondence," Commun. Math. Phys. 217, 595 (2001) arXiv:hep-th/0002230.

[27] M. Bianchi, D. Z. Freedman and K. Skenderis, "How to go with an RG flow," JHEP 0108, 041 (2001) arXiv:hep-th/0105276.

[28] M. Bianchi, D. Z. Freedman and K. Skenderis, "Holographic renormalization," Nucl. Phys. B 631, 159 (2002) arXiv:hep-th/0112119.

[29] I. Papadimitriou and K. Skenderis, "Thermodynamics of asymptotically locally AdS spacetimes," JHEP 0508, 004 (2005) arXiv:hep-th/0505190.

[30] A. Parnachev and A. Starinets, "The silence of the little strings," arXiv:hep-th/0506144.

[31] S. W. Hawking and G. T. Horowitz, "The Gravitational Hamiltonian, action, entropy and surface terms," Class. Quant. Grav. 13, 1487 (1996) arXiv:gr-qc/9501014.

[32] A. Buchel and L. A. Pando Zayas, "Hagedorn vs. Hawking-Page transition in string theory," Phys. Rev. D 68, 066012 (2003) arXiv:hep-th/0305179.

[33] A. Buchel and J. T. Liu, "Thermodynamics of the $\mathrm{N}=2^{*}$ flow," JHEP 0311, 031 (2003) arXiv:hep-th/0305064.

[34] A. Buchel, "N $=2^{*}$ hydrodynamics," Nucl. Phys. B 708, 451 (2005) arXiv:hep-th/0406200.

[35] G. Policastro, D. T. Son and A. O. Starinets, "From AdS/CFT correspondence to hydrodynamics. II: Sound waves," JHEP 0212, 054 (2002) arXiv:hep-th/0210220.

[36] P. Kovtun, D. T. Son and A. O. Starinets, "Holography and hydrodynamics: Diffusion on stretched horizons," JHEP 0310, 064 (2003) arXiv:hep-th/0309213. 
[37] A. Buchel and J. T. Liu, "Universality of the shear viscosity in supergravity," Phys. Rev. Lett. 93, 090602 (2004) arXiv:hep-th/0311175.

[38] P. Kovtun, D. T. Son and A. O. Starinets, "Viscosity in strongly interacting quantum field theories from black hole physics," Phys. Rev. Lett. 94, 111601 (2005) arXiv:hep-th/0405231.

[39] A. Buchel, "On universality of stress-energy tensor correlation functions in supergravity," Phys. Lett. B 609, 392 (2005) arXiv:hep-th/0408095. 